\title{
Desarrollo de una herramienta para la evaluación de la calidad percibida en los centros de atención infantil temprana
}

\author{
Rita-Pilar Romero-Galisteo ${ }^{1 *}$, Verónica Morales-Sánchez ${ }^{2}$ y Antonio Hernández-Mendo ${ }^{2}$ \\ 1 Centro de Atención Infantil Temprana del Excmo. Ayto. de Antequera (Málaga) y Universidad de Málaga. Dpto. de Psiquiatría y Fisioterapia. Facultad de CC de la Salud \\ 2 Universidad de Málaga. Dpto. de Psicología Social, Antropología Social, Trabajo Social y Servicios Sociales. Facultad de Psicología
}

Resumen: Este trabajo describe el desarrollo de un instrumento para evaluar la calidad percibida en los Centros de Atención Infantil Temprana (CAIT). La muestra estuvo constituida por 672 usuarios/as (508 mujeres y 162 hombres) de entre 20 y 60 años, de 14 de los 16 CAIT que existían en la provincia de Málaga en el momento de la recogida de los datos. Los resultados del análisis factorial exploratorio evidencian una estructura de diez factores y unos índices de consistencia interna satisfactorios. Se concluye que la herramienta desarrollada, denominada Inventario de Calidad en los Centros de Atención Infantil Temprana (ICCAIT), constituye un instrumento con importantes evidencias de validez y fiabilidad que permite evaluar, especialmente en CAIT, la calidad percibida por parte de los/as usuarios/as de los mismos.

Palabras clave: Atención temprana; calidad percibida; calidad de servicio; familia.

\section{Introducción}

Durante los últimos 30 años, en España se ha producido una paulatina pero sólida integración de la Atención Temprana (AT). Actualmente puede decirse que es una realidad suficientemente consolidada después de más de tres décadas de trabajo con familias y niños con trastornos del desarrollo o riesgo de padecerlos (Giné, Gràcia, Vilaseca y García-Díe, 2006). Ha de considerarse que la AT en nuestro país ha experimentado un gran progreso en los últimos 20 años, se han dado cambios a nivel administrativo, legislativo, en la estructura, en la organización y en la financiación. El concepto, la filosofía y los diferentes modelos han ido adaptándose a las necesidades de los niños y de sus familias, en ocasiones, aunque no siempre, con la colaboración entre los estamentos gubernamentales, responsables de las políticas sociales, sanitarias y educativas y los profesionales del sector, unidos con las Asociaciones y Federaciones de familias de niños con discapacidad (Grupo de Atención Temprana [GAT], 2011).

La AT ha sufrido también un cambio de orientación desde modelos centrados en el niño al modelo centrado en la familia (Iversen, Shimmed, Ciacera y Prabhakar, 2003). El modelo de servicio centrado en la familia es una filosofía y un método de provisión de los servicios que enfatiza la colaboración entre padres y profesionales, implicando a la familia en la toma de decisiones y contando con su opinión (Medina i Mirapeix, De Oliveira, Lillo, Montilla, Escolar y Gómez, 2007; Pegenaute, 2009; Romero-Galisteo y Morales-Sánchez, 2010a, b).

* Dirección para correspondencia [Correspondence address]: Rita Pilar Romero Galisteo. Universidad de Málaga. Dpto. de Psiquiatría y Fisioterapia. Facultad Ciencias de la Salud. C/ Arquitecto Francisco Peñalosa s/n. Ampliación del Campus de Teatinos. 29071 Málaga (España). E-mail: rpromero@uma.es / rromegal@gmail.com
Title: Development of a tool for assessing the perceived quality in Early Intervention Centres.

Abstract: This paper describes the development of an instrument to assess the perceived quality in Early Intervention Centers (CAIT). The sample consisted of 672 users (508 women and 162 men) aged between 20 and 60 years, from 14 of the 16 centres that existed in the province of Malaga at the time of data collection. The exploratory factor analysis results shows a structure of ten factors. The obtained results are satisfactory in reliability. We conclude that the developed tool called Quality Inventory in Early Intervention Centres (ICCAIT), is an instrument with strong evidence of validity and reliability that allows evaluation, especially in CAIT, the quality perceived by users same.

Key words: Early intervention; perceived quality; quality of service; family.

De forma paralela, la interdisciplinariedad se ha convertido en la característica que define la actividad científica del S. XXI (Kessel \& Rosenfield, 2008). Este trabajo confiere importancia a la confluencia de las ciencias biológicas y sociales en la investigación sobre AT. Este tipo de estudio, por definición, implica conocimiento de técnicas y experiencias de diferentes disciplinas (Shonkoff \& Phillips, 2000). La investigación interdisciplinar es un proceso que responde a una pregunta, resuelve un problema o trata un tema demasiado amplio o complejo para ser tratado adecuadamente por una sola disciplina o profesión. Se ha de basar en puntos de vista diferentes y debe integrar sus distintas perspectivas de un modo más amplio. Hablar de disciplinas diferentes implica tratar de forma diferente realidades que hacen referencia al uso del término calidad de servicio.

La calidad es uno de los elementos estratégicos en que se fundamenta la transformación y la mejora de los sistemas organizativos modernos. En toda clase de organizaciones vinculadas al sector servicios, cuando se analiza la calidad se obtiene que, a pesar de sus potenciales beneficios, la implantación de una estrategia competitiva de esta naturaleza no está exenta de problemas, habida cuenta de la dificultad que plantea la definición y medida de la misma en el ámbito de la salud. Pese a ello, son varios los investigadores que han tratado de desarrollar definiciones y diseñar modelos para gestionar adecuadamente la calidad (Urbina-Pérez, Pérez-Pérez y Martínez-Sánchez, 2000; Morales-Sánchez, 2011).

Según Kotler (2000), los servicios centrados en el cliente se encuentran en mejor posición para identificar nuevas oportunidades y establecer estrategias que proporcionen beneficios a largo plazo. Las empresas deben establecer sus objetivos priorizando sobre los clientes. Para esto, las estrategias que se definen estarán encaminadas a satisfacerlos, haciendo hincapié en la mejora continua de los procesos, orientándolos a satisfacer las necesidades de estos clientes. Para 
ello, deben adaptar y orientar continuamente los recursos y la organización a dichos clientes. De esta manera podrá decidirse qué necesidades emergentes son más importantes.

La AT no puede ser ajena a la expansión de los sistemas de calidad en el sector servicios, en los que la satisfacción del cliente es siempre una clave fundamental. En AT la satisfacción del cliente-familia resulta, si cabe, especialmente trascendente, por el papel multidimensional que la familia juega en esta disciplina y por la subjetividad que le es propia por el momento que está viviendo. Por este motivo, ha de ser analizada la valoración de la satisfacción de las familias en este campo, contemplando diferentes dimensiones (García, Mirete, Marín y Romero, 2008).

Actualmente, cualquier acción sobre las personas no se entiende sin tener una medida de calidad del proceso de intervención y de la calidad percibida por los/as usuarios/as y su familia. Pese a los sistemas de acreditación o normalización, los servicios de AT están necesitados de una herramienta pragmática que permita conocer la opinión de sus clientes (Pegenaute, 2009). Teniendo en cuenta la importancia y contribución de la familia para la promoción del desarrollo de los menores, en especial aquellos que se encuentran en situación de riesgo o que presentan algún tipo de discapacidad, es fundamental que las estrategias utilizadas por los profesionales incluyan a la familia y no, simplemente a los niños (Cerqueira da Silva, Dessen y Pérez-López, 2012; Pereira-Silva y Dessen, 2005; Pérez-López y Sánchez-Caravaca, 2008). Una vez determinada esta necesidad, la tarea adicional corresponde el tratar de establecer cuáles pueden ser los contenidos mínimos que se necesitan para evaluar los servicios de AT en general y los centros de atención temprana en particular, objeto de este trabajo (Romero-Galisteo, MoralesSánchez y Sánchez-Guerrero, 2011).

Una vez establecidas y consolidadas las bases de la AT en España, entendida como se la define en el Libro Blanco de la AT (GAT, 2000), es decir, como el conjunto de intervenciones, dirigidas a la población infantil de cero a seis años, a la familia y al entorno, que tiene por objetivo dar respuesta lo más pronto posible a las necesidades transitorias o permanentes que presentan los niños con trastornos en su desarrollo o que tienen el riesgo de padecerlos; se antojan necesarias otro tipo de investigaciones, ya que, como apuntan PérezLópez et al. (2012) y Trianes et al. (2009), actualmente en la evaluación infantil y en AT se produce una demanda de instrumentos que sean óptimos para la recolección de datos sistemáticos y representativos.

Los Centros de Atención Infantil Temprana, en adelante CAIT, se encargan de atender a la población infantil que tiene un trastorno del desarrollo ya establecido o un alto riesgo de desarrollarlo, a través de una intervención profesional especializada dirigida a potenciar capacidades, evitando, o en su caso minimizando, el agravamiento de una posible discapacidad. Los CDIAT (Centros de Desarrollo Infantil y Atención Temprana) o CAIT, como se denominan en Andalucía, son servicios autónomos, de carácter interdisciplinar, que surgieron en España alrededor de los años 70 y han experi- mentado una constante evolución a lo largo de su trayectoria histórica, adecuándose a las crecientes demandas de atención por parte de la población infantil y de sus familias. Este proceso ha estado motivado por la mejor detección y diagnóstico de los distintos trastornos del desarrollo, y por el reconocimiento de su origen multifactorial, factores ambos que han destacado la importancia capital de una intervención temprana (Milla y Mulas, 2002).

La creación y evolución de los CDIAT/CAIT ha sido paralela a la existencia y orientación de otros servicios e instituciones que desde los ámbitos sanitario, social y educativo en las respectivas comunidades autónomas, han dado y dan respuestas a determinados colectivos o a necesidades específicas de los mismos. Esta adecuación, y la diversa dependencia administrativa, ha generado diferentes modelos de CDIAT/CAIT (Bugié, 2002). En determinadas comunidades o zonas geográficas, en función de su historia y recursos asistenciales, algunos colectivos de niños con trastornos específicos en su desarrollo son atendidos bien en CDIAT/CAIT o en programas o unidades de AT que están articulados con otros servicios o centros. Así, encontramos en Málaga y su provincia centros de diversa índole, la mayor parte evaluados en este trabajo, que comprenden desde asociaciones de padres, colegios, centros municipales, privados, etc.

Expuesta la situación de la que partió esta investigación, se propuso construir un cuestionario que tuviera como objeto la evaluación de la calidad percibida en los CAIT. Como paso previo y necesario, fue comprobada la carencia e inadecuación de los instrumentos existentes para tal fin. Consultando las referencias se evidenció que en España no se cuenta con un instrumento que evalúe la percepción de la calidad en los CAIT por parte de las familias - usuarios/as atendidas en dichos centros. Fueron identificados diversos instrumentos que tenían como objetivo central, bien evaluar la satisfacción del cliente-familia (García et al., 2008), bien analizar la percepción de los padres, pero únicamente de la atención de Fisioterapia recibida en los CAIT (Medina i Mirapeix et al., 2007). A pesar de comprobar que estos instrumentos se apoyaban en una sólida base teórica, se alejaban considerablemente de nuestra fundamentación teórica de partida, consistente en la evaluación de la calidad percibida por parte de las familias atendidas en los CAIT y más concretamente en aquellos situados en Málaga y su provincia. Se probó entonces que los instrumentos analizados no cubrían esta necesidad.

Dada la inexistencia de instrumentos válidos que tuvieran como objetivo principal evaluar la calidad percibida en los CAIT o que cumplieran con las propiedades psicométricas requeridas, se optó por construir un cuestionario que diera respuesta a dicha carencia y estuviera destinado a medir la calidad del servicio en este tipo de centros. Para la definición semántica del constructo se realizó una revisión de los trabajos existentes sobre atención temprana y calidad de servicio (Arizcun, Gútiez y Ruiz, 2004; Barbarin y Waslk, 2009; García-Sánchez, Castellanos y Mendieta, 1998; García-Sánchez, 2002; GAT, 2000, 2005; Morales-Sánchez, 2003; Morales- 
Sánchez, Hernández-Mendo y Blanco, 2009: Pegenaute, 2009; Peréz-López y Brito de la Nuez, 2004; Perpiñán, 2009; Pina, 2008; Ponte, 2003a,b; Ponte et al., 2004; Shonkoff y Levitt, 2010; Vanderven, 2008; Vegas y Santibáñez, 2010). El cuestionario quedó definido de acuerdo con aquellas dimensiones consideradas clave para la evaluación de la calidad de servicio y más concretamente de los servicios de atención temprana: las instalaciones del centro, las salas de tratamiento y material utilizado, la atención al usuario, la información relativa a los profesionales del centro o a la información general y técnica, que engloban tanto aspectos tangibles como intangibles del servicio.

\section{Método}

\section{Participantes}

La obtención de la muestra de usuarios/as se realizó utilizando un muestreo de conglomerados. En Andalucía, en esta temática, la situación es heterogénea y compleja ya que actividades de AT se llevan a cabo en centros educativos, centros de servicios sociales especializados, centros sanitarios públicos, centros privados concertados, centros de entidades sin ánimo de lucro y centros dependientes de corporaciones locales, aunque en definitiva todos pueden considerarse CAIT por lo que la muestra de participantes es representativa. En el momento de la recogida de datos, existían en Andalucía 93 equipos de intervención, 16 de los cuales se ubicaban en la provincia de Málaga, participando en esta investigación 14 de los mismos.

Tanto en el estudio piloto como en el estudio final, la muestra de participantes fueron los padres y madres de los/as niños/as que reciben tratamiento de AT en los CAIT que colaboraron en el estudio. En el caso del estudio piloto, se utilizaron tres centros pertenecientes a la ciudad de Málaga y se recogieron 102 encuestas, mientras que para el estudio final, se utilizaron catorce de los dieciséis centros con los que hasta la fecha de la recogida de datos contaba la provincia de Málaga y se recogieron un total de 672 encuestas.

La muestra de usuarios/as participantes en el estudio piloto fue mayoritariamente de género femenino, siendo 72 (70.6\%) el número de madres que cumplimentaron el cuestionario y 29 padres (28.4\%), cuyo rango de edad oscila entre los 20 y los 60 años. El porcentaje mayor (12.7\%) corresponde a los 34 años, con un total de 13 usuarios/as con esa edad.

En cuanto al estudio final, la muestra se compone de 508 mujeres $(75.6 \%)$ y 162 hombres $(24.1 \%)$ con edades comprendidas entre los 20 y los 80 años, siendo el intervalo entre los 30 y 40 años $(D T=.84)$ el que más se repite y constituyendo el $58.3 \%$ de la muestra. Asimismo, resultaba interesante recoger y analizar el parentesco de los participantes con los/as usuarios/as directos del servicio, es decir, con los niños y niñas atendidos en el mismo. Son las madres las que con un $72.3 \%$ (486) han contestado mayoritariamente al cuestionario. La distribución de participantes por centro se observa en la Tabla 1.

Tabla 1. Participantes por centro

\begin{tabular}{|c|c|c|}
\hline $\begin{array}{l}\text { CENTROS DE ATENCIÓN } \\
\text { INFANTIL TEMPRANA }\end{array}$ & $\begin{array}{c}\text { ICCAIT-v.1.0 } \\
N^{\circ} \text { DE } \\
\text { USUARIOS }\end{array}$ & $\begin{array}{c}\text { ICCAIT-v.2.0 } \\
N^{\circ} \text { DE } \\
\text { USUARIOS }\end{array}$ \\
\hline ASPAHIDEV & $53(52 \%)$ & $42(6.25 \%)$ \\
\hline La Purísima & $16(15.7 \%)$ & $24(3.57 \%)$ \\
\hline Amigos Especiales de Coín & & $9(1.33 \%)$ \\
\hline АМАРPACE & & $45(6.69 \%)$ \\
\hline Vírgen de la Esperanza & & $54(8.03 \%)$ \\
\hline Antequera & & $70(10.41 \%)$ \\
\hline Equipo SIDI & $33(32.3 \%)$ & $36(5.35 \%)$ \\
\hline ASPANDEM & & $67(9.97 \%)$ \\
\hline FAHALA & & $74(11.01 \%)$ \\
\hline Vélez-Málaga & & $91(13.54 \%)$ \\
\hline Autismo Málaga & & $10(1.48 \%)$ \\
\hline Taller de la Amistad & & $29(4.31 \%)$ \\
\hline APRONA & & $89(13.24 \%)$ \\
\hline ASPRODISIS & & $32(4.76 \%)$ \\
\hline TOTAL & $102(100 \%)$ & $672(100 \%)$ \\
\hline
\end{tabular}

\section{Instrumento}

Para la construcción de la herramienta se partió de los estudios realizados por Medina i Mirapeix et al. (2007) con el Cuestionario de Calidad Percibida en la Fisioterapia de Atención Temprana y de García et al. (2008) con el Cuestionario sobre Satisfacción del Cliente Familia. Asimismo, se tuvieron en cuenta tanto las directrices de Muñiz, Fidalgo, GarcíaCueto, Martínez y Moreno (2005) como las teorías sobre la calidad de Donabedian (1988), uno de los más importantes teóricos sobre la calidad de la atención en la salud, al igual que las aportaciones realizadas por Arias-Astray y Fernández-Ramírez (1998) y Anguera (2003).

De esta forma la versión preliminar del Inventario de Calidad para Centros de Atención Infantil Temprana, denominado ICCAIT-v.1.0, incluyó 63 ítems que fueron sometidos a un proceso de depuración que incluye, en primer lugar, un comité de expertos siguiendo así las recomendaciones de Abad, Olea, Ponsoda y García (2011), y en segundo lugar, un estudio piloto que incluyó un análisis de fiabilidad y un análisis factorial para comprobar las propiedades métricas de los ítems.

La segunda versión de la herramienta (ICCAIT-v.2.0), se compone de 48 ítems agrupados en 6 escalas: instalaciones del centro, salas de tratamiento y material, atención al usuario, personal especializado, información general e información técnica. Las respuestas se recogen en un continuo de cinco puntos, en el que 1 significa nada de acuerdo y 5 muy de acuerdo con el enunciado del ítem. Por último, se consideró importante la recogida de datos sociodemográficos y se añadió un apartado de observaciones y/o sugerencias, cuya información será objeto de futuros análisis. En el apéndice se encuentra el ICCAIT-v.2.0. 


\section{Procedimiento}

El estudio piloto sirvió para perfilar nuestro cuestionario inicial, al que denominamos ICCAIT-v.1.0. Posteriormente, ampliamos la muestra tanto de participantes como de centros colaboradores utilizando la segunda versión de la herramienta (ICCAIT-v.2.0), más pragmática y reducida en cuanto a número de ítems que la anterior, realizando nuevos análisis con los datos recogidos.

Previo consentimiento y aceptación de colaboración de los diferentes centros participantes, tanto la primera versión del cuestionario (ICCAIT-v.1.0) como la segunda versión (ICCAIT-v.2.0), fueron administradas en horario de atención de los distintos servicios de AT por 37 encuestadores debidamente formados para tal fin. En las instrucciones a los/as participantes se indicó que la colaboración en el estudio era voluntaria, prestando especial atención a la confidencialidad y anonimato de los datos proporcionados. La cumplimentación del cuestionario se realizó de manera individual por el familiar que acompañaba en ese momento al niño/a a la sesión de tratamiento correspondiente. En la mayoría de los centros, se llevó a cabo en la sala de espera de los mismos, es decir, sin la presencia, posiblemente coartadora, de cualquiera de los profesionales que integraban los equipos de los distintos CAIT participantes. Los encuestadores estuvieron presentes en todo momento para solucionar posibles dudas en la comprensión de los ítems, caso que se presentó en mínimas ocasiones. Acto seguido, se numeraban los cuestionarios y en ningún momento se mostraron al personal de los centros participantes. El tiempo aproximado de contestación fue de quince minutos.

\section{Análisis de datos}

Una de las técnicas más comunes para determinar la estructura subyacente de un cuestionario es el AFE, que consiste en resumir la información contenida en una matriz de datos de variables identificando un reducido número de factores menor que el número de variables. Por tanto, si no se posee una concepción previa de la estructura del constructo, como en nuestro caso, el uso del $A F E$ es adecuado para purificar los datos, y contribuye a la clarificación conceptual y desarrollo de mejores instrumentos de medida (TomásMiguel, 1993).

Así, con objeto aportar en este estudio evidencias de validez de constructo y de disminuir el número inicial de ítems, optimizando así la herramienta creada, se llevó a cabo un análisis factorial exploratorio $(A F E)$, utilizándose el método de componentes principales con rotación varimax, para obtener un reparto más homogéneo de la varianza explicada con una estructura más fácil de interpretar (Abad et al., 2011). Los criterios de pertinencia considerados para la realización del $A F E$ fueron el determinante de la matriz, la medida de adecuación de Kaiser-Meyer-Olkin (KMO) y el test de esfericidad de Bartlett. En la eliminación de los ítems, se utilizó como criterio los resultados del alpha de Cronbach si se elimina el elemento en el análisis de consistencia interna, mejorando de esta manera tanto la fiabilidad como los índices $K M O$ y la varianza explicada de cada dimensión. Para el análisis de datos se utilizó el paquete estadístico SPSS-v.15.0.

\section{Resultados}

Se muestran los estadísticos descriptivos de los ítems del ICCAIT-v.2.0 referidos a media, desviación típica, asimetría y curtosis. Los elementos mejor valorados se centran en el personal especializado, concretamente la cercanía en el trato $(M=4.85)$, la accesibilidad $(M=4.82)$ así como los conocimientos y las aportaciones e iniciativas $(M=4.78)$. Por el contrario, la dimensión relativa a la información general cuenta con los dos ítems peor valorados con medias de 1.81 (38) y 2.22 (39) respectivamente. Respecto a la normalidad de la muestra, existe cierto grado de asimetría negativa con excepción de los ítems 38 y 39, mientras que la curtosis refleja una marcada distribución platicúrtica, rechazando de esta manera la hipótesis de normalidad multivariante (Tabla 2).

Tabla 2. Media $(M)$, desviación típica $(D T)$ asimetría y curtosis.

\begin{tabular}{|c|c|c|c|c|c|}
\hline Escala & Ítem & $M$ & $D T$ & Asimetría & Curtosis \\
\hline \multirow[t]{13}{*}{1} & 1 & 4.08 & 1.128 & -1.152 & .590 \\
\hline & 2 & 3.41 & 1.423 & -.439 & -.972 \\
\hline & 3 & 3.34 & 1.475 & -.346 & -1.219 \\
\hline & 4 & 4.39 & 1.068 & -1.962 & 3.242 \\
\hline & 5 & 4.67 & .711 & -2.759 & 8.925 \\
\hline & 6 & 4.57 & .744 & -1.897 & 3.898 \\
\hline & 7 & 4.26 & 1.046 & -1.556 & 1.951 \\
\hline & 8 & 4.06 & 1.237 & -1.307 & .792 \\
\hline & 9 & 4.32 & 1.082 & -1.685 & 2.160 \\
\hline & 10 & 4.59 & .878 & -2.495 & 6.188 \\
\hline & 11 & 4.11 & 1.117 & -1.151 & .457 \\
\hline & 12 & 3.92 & 1.232 & -1.017 & .160 \\
\hline & 13 & 3.06 & 1.572 & -.117 & -1.507 \\
\hline \multirow[t]{9}{*}{2} & 14 & 4.04 & 1.156 & -1.115 & .423 \\
\hline & 15 & 4.24 & 1.026 & -1.475 & 1.894 \\
\hline & 16 & 4.20 & 1.058 & -1.453 & 1.781 \\
\hline & 17 & 4.22 & 1.030 & -1.486 & 2.092 \\
\hline & 18 & 4.54 & .758 & -1.999 & 4.947 \\
\hline & 19 & 4.42 & .893 & -1.811 & 3.687 \\
\hline & 20 & 4.48 & .811 & -1.935 & 5.039 \\
\hline & 21 & 4.62 & .687 & -2.394 & 8.436 \\
\hline & 22 & 4.58 & .772 & -2.616 & 9.660 \\
\hline \multirow[t]{7}{*}{3} & 23 & 4.75 & .586 & -2.869 & 10.113 \\
\hline & 24 & 3.44 & 1.444 & -.439 & -1.141 \\
\hline & 25 & 4.14 & 1.188 & -1.349 & .872 \\
\hline & 26 & 4.22 & 1.179 & -1.452 & 1.149 \\
\hline & 27 & 4.73 & .663 & -3.211 & 12.619 \\
\hline & 28 & 4.03 & 1.184 & -1.142 & .545 \\
\hline & 29 & 4.64 & .755 & -2.550 & 7.187 \\
\hline \multirow[t]{8}{*}{4} & 30 & 4.78 & .590 & -3.531 & 15.150 \\
\hline & 31 & 4.82 & .498 & -3.380 & 14.528 \\
\hline & 32 & 4.77 & .580 & -3.152 & 11.790 \\
\hline & 33 & 4.85 & .475 & -4.415 & 24.788 \\
\hline & 34 & 4.82 & .519 & -3.613 & 15.627 \\
\hline & 35 & 4.78 & .554 & -3.423 & 15.170 \\
\hline & 36 & 4.68 & .706 & -2.831 & 9.605 \\
\hline & 37 & 4.70 & .733 & -3.526 & 15.329 \\
\hline
\end{tabular}




\begin{tabular}{lccccc}
\hline Escala & Ítem & $M$ & $D T$ & Asimetría & Curtosis \\
\hline 5 & 38 & 1.81 & 1.464 & 1.442 & .395 \\
& 39 & 2.22 & 1.703 & .792 & -1.164 \\
& 40 & 3.30 & 1.738 & -.356 & -1.568 \\
& 41 & 4.27 & 1.217 & -1.634 & 1.764 \\
6 & 42 & 3.57 & 1.628 & -.660 & -1.137 \\
& 43 & 4.60 & .766 & -2.290 & 5.824 \\
& 44 & 4.63 & .739 & -2.490 & 7.116 \\
& 45 & 4.61 & .778 & -2.520 & 7.262 \\
& 46 & 4.54 & .968 & -2.467 & 5.837 \\
& 47 & 4.34 & 1.171 & -1.825 & 2.298 \\
& 48 & 4.66 & .782 & -2.815 & 8.307 \\
\hline
\end{tabular}

En el análisis factorial exploratorio, los valores iniciales obtenidos por escalas (Tabla 3) para los criterios de pertinencia mostraron la adecuación para su realización. Así, el determinante de la matriz de correlaciones resulta satisfactorio con valores próximos a 0 en todas las escalas (Martínez, 1996; Visauta, Martori y Cañas, 2005). Asimismo los valores del índice KMO oscilan entre .528 y .888 y el test de esfericidad de Bartlett presenta valores significativos $(p<.005)$ consiguiéndose además valores altos de $\chi^{2}$, por lo que las variables presentan altas correlaciones.

Tabla 3. Resultados del AFE del cuestionario ICCAIT-v.2.0

\begin{tabular}{|c|c|c|c|}
\hline Descriptivos & Determinante & KMO & $\begin{array}{c}\text { Test esfericidad de } \\
\text { Bartlett }\end{array}$ \\
\hline $\begin{array}{l}\text { Escala } 1 \text { (ítems del } \\
1 \text { al 13) }\end{array}$ & .022 & .868 & $\begin{array}{c}\chi^{2}: 2506.401 \\
\text { gl: } 78 \\
\text { sig: } .000\end{array}$ \\
\hline $\begin{array}{l}\text { Escala } 2 \text { (ítems del } \\
14 \text { al 22) }\end{array}$ & .013 & .888 & $\begin{array}{c}\chi^{2:}: 2854.960 \\
\text { gl: } 36 \\
\text { sig: } .000\end{array}$ \\
\hline $\begin{array}{l}\text { Escala } 3 \text { (ítems del } \\
23 \text { al 29) }\end{array}$ & .218 & .790 & $\begin{array}{c}\chi^{2}: 1005.171 \\
\text { gl: } 21 \\
\text { sig: } .000\end{array}$ \\
\hline $\begin{array}{l}\text { Escala } 4 \text { (ítems del } \\
\text { 30al 37) }\end{array}$ & .048 & .886 & $\begin{array}{c}\chi^{2}: 2013.523 \\
\text { gl: } 28 \\
\text { sig: } .000\end{array}$ \\
\hline $\begin{array}{l}\text { Escala } 5 \text { (ítems del } \\
38 \text { al 42) }\end{array}$ & .868 & .528 & $\begin{array}{c}\chi^{2:}: 93.864 \\
\text { gl: } 10 \\
\text { sig: } .000\end{array}$ \\
\hline $\begin{array}{l}\text { Escala } 6 \text { (ítems del } \\
43 \text { al 48) }\end{array}$ & .062 & .874 & $\begin{array}{c}\chi^{2}: 1857.771 \\
\text { gl: } 15 \\
\text { sig: } .000\end{array}$ \\
\hline
\end{tabular}

Se observó además que, tanto los valores iniciales del indice KMO como del \% de varianza explicada podían mejorarse, optimizando así la herramienta propuesta. Esta optimización se llevó a cabo de la manera explicada anteriormente en el apartado de análisis de datos. La mejora de los nuevos valores obtenidos puede comprobarse en la comparativa que se muestra en la Tabla 4 en la que se observan valores finales que oscilan entre el 49.417 y el $69.850 \%$ de la varianza total explicada y entre el .528 y el .885 del KMO.
Tabla 4. Resultados comparativos entre el ICCAT-v.2.0 y su optimización

\begin{tabular}{lcccc}
\hline Escala & $\begin{array}{c}\text { KMO } \\
\text { inicial }\end{array}$ & $\begin{array}{c}\% \text { Varianza } \\
\text { inicial }\end{array}$ & $\begin{array}{c}\text { KMO } \\
\text { final }\end{array}$ & $\begin{array}{c}\% \text { Varianza } \\
\text { final }\end{array}$ \\
\hline 1 & .868 & 54.06 & .868 & 57.875 \\
2 & .888 & 63.085 & .885 & 69.850 \\
3 & .790 & 41.673 & .790 & 41.673 \\
4 & .886 & 50.011 & .884 & 56.494 \\
5 & .528 & 49.975 & .528 & 49.975 \\
6 & .874 & 61.417 & .874 & 61.417 \\
\hline
\end{tabular}

Partiendo del conjunto de 48 ítems que habían superado los criterios pertinentes y con el fin de explorar la validez de constructo, el paso siguiente fue analizar de qué manera se agrupaban los ítems. La solución factorial definitiva del cuestionario a partir de los 48 ítems se realizó mediante el mismo procedimiento expuesto en párrafos anteriores y observando las matrices de componentes rotados de cada escala para ver en cuántos factores saturaba cada uno. De esta manera obtuvimos una solución factorial de 10 factores, como puede observarse en la Tabla 5, a los que hemos denominado: sala de espera; condiciones ambientales del centro; ubicación del centro; material; salas de tratamiento; atención al usuario; cualificación, coordinación y cercanía del personal al usuario; derivación al centro; sugerencias, reclamaciones y quejas e información técnica.

Tabla 5. Estructura factorial del ICCAIT-v.2.0

\begin{tabular}{|c|c|c|c|}
\hline Escala & Items & Denominación & $\begin{array}{l}\% \mathrm{Va}- \\
\text { rianza }\end{array}$ \\
\hline \multirow[t]{3}{*}{1} & $8,10,11,12,13$ & Sala de espera & 35.489 \\
\hline & $4,5,6,7,9$ & $\begin{array}{l}\text { Condiciones ambientales } \\
\text { del centro }\end{array}$ & 10.032 \\
\hline & $1,2,3$ & Ubicación del centro & 8.546 \\
\hline \multirow[t]{2}{*}{2} & $18,19,20,21,22$ & Material & 51.730 \\
\hline & $14,15,16,17$ & Salas de tratamiento & 11.354 \\
\hline 3 & $\begin{array}{l}23,24,25,26, \\
27,28,29\end{array}$ & Atención al usuario & 41.673 \\
\hline 4 & $\begin{array}{l}30,31,32,33 \\
34,35,36,37\end{array}$ & $\begin{array}{l}\text { Cualificación, coordina- } \\
\text { ción, cercanía del personal }\end{array}$ & 50.011 \\
\hline \multirow[t]{2}{*}{5} & 38,39 & Derivación al centro & 27.813 \\
\hline & $40,41,42$ & $\begin{array}{l}\text { Sugerencias, reclamaciones } \\
\text { y quejas }\end{array}$ & 22.163 \\
\hline 6 & $\begin{array}{l}43,44,45,46, \\
47,48\end{array}$ & Información técnica & 61.417 \\
\hline
\end{tabular}

Con la finalidad de explorar la existencia de diferencias significativas en función del género se procedió a realizar un análisis descriptivo calculando las medias y desviaciones típicas, así como un análisis de comparación de medias t-test entre los participantes. Como se puede observar en la Tabla 6, en los factores ubicación del centro, salas de tratamiento y cualificación, coordinación y cercanía del personal se aprecian diferencias significativas entre hombres y mujeres, siendo estas últimas las que señalan medias más altas en la puntuación de dichas dimensiones. Sin embargo, en el resto de los factores estas diferencias no son significativas. 
Tabla 6. Medias, desviaciones típicas y resultados del t-test para los factores en función del género

\begin{tabular}{|c|c|c|c|c|c|}
\hline \multirow[t]{2}{*}{ Factor } & \multicolumn{2}{|c|}{$\begin{array}{l}\text { Hombres } \\
(n=162)\end{array}$} & \multicolumn{2}{|c|}{$\begin{array}{l}\text { Mujeres } \\
(n=510)\end{array}$} & \multirow[t]{2}{*}{ t-test } \\
\hline & $M$ & DT & $M$ & $D T$ & \\
\hline Sala de espera & 3.89 & 0.84 & 3.97 & 0.84 & 0.31 \\
\hline Condiciones ambientales del centro & 4.40 & 0.65 & 4.45 & 0.63 & 0.39 \\
\hline Ubicación del centro & 3.40 & 1.04 & 3.67 & 1.00 & $0.00^{*}$ \\
\hline Material & 4.53 & 1.02 & 4.54 & 0.64 & 0.82 \\
\hline Salas de tratamiento & 4.05 & 0.87 & 4.21 & 0.86 & $0.03^{*}$ \\
\hline Atención al usuario & 4.27 & 0.70 & 4.29 & 0.63 & 0.23 \\
\hline $\begin{array}{l}\text { Cualificación, coordinación, cercanía } \\
\text { del personal }\end{array}$ & 4.70 & 0.53 & 4.80 & 0.48 & $0.01 *$ \\
\hline Derivación al centro & 2.11 & 1.18 & 1.97 & 1.21 & 0.18 \\
\hline Sugerencias, reclamaciones y quejas & 3.75 & 0.94 & 3.70 & 1.01 & 0.57 \\
\hline Información técnica & 4.53 & 0.66 & 4.57 & 0.67 & 0.50 \\
\hline
\end{tabular}

La consistencia interna de la versión final del cuestionario fue estimada a partir de la totalidad de la muestra y mediante el coeficiente alpha de Cronbach. Los resultados mostraron una buena fiabilidad en todas las escalas salvo en la 5, siguiendo el rango establecido por Nunnally (1976). Se obtiene un $\alpha=$ .829 para la primera escala; $\alpha=.885$ para la segunda escala; $\alpha=.738$ para la tercera escala; $\alpha=.861$ para la cuarta escala; $\alpha=.322$ para la quinta escala y $\alpha=.860$ para la sexta escala. Aunque la cuantía del coeficiente $\alpha$ para la quinta escala pudiera considerarse bajo, conviene recordar como apuntan Abad et al. (2011) que el número de ítems es uno de los factores que influyen en el valor de $\alpha$ y que la quinta escala está constituida por tan solo 5 ítems, por lo que puede ser aceptado.

\section{Discusión y Conclusiones}

Los resultados obtenidos en este estudio avalan tanto la adecuación de las propiedades psicométricas de los ítems como la validez y fiabilidad del Inventario de Calidad en los Centros de Atención Infantil Temprana. Los diferentes análisis realizados aportan evidencia de la validez de una estructura de diez factores acorde con los planteamientos teóricos previos a la vez que muestran unos índices estadísticos adecuados (Tabachnick y Fidell, 2007). De igual manera, merece destacar y valorar la evidencia y buenos índices de consistencia interna obtenidos para el conjunto de escalas que componen el cuestionario (Clark y Watson, 1995).

Consideramos que el ICCAIT es un instrumento que proporciona información útil tanto por áreas como por asuntos específicos. Permite además, identificar problemas específicos relacionados con la percepción de la calidad y resulta muy operativo para encontrar vías de acción cuando se quiere actuar sobre algunas de las causas que pueden provocar una negativa valoración global de la calidad del centro o de los profesionales del mismo. Cabe mencionar que la cultura de la calidad resulta exitosa cuando todos los integrantes de la organización demuestran capacidad para trabajar en equipo. La planificación, el control y la mejora de los procesos con los que se trabaja e interactúa mediante el uso de metodologías ad hoc resultan siempre adecuados para instaurar mejoras en la gestión de la calidad. Mostrar hábitos, valores y actitud de servicio hacia los clientes internos y externos, a quienes se tratará siempre de satisfacer, son estrategias a tener en cuenta a la hora de aplicar métodos de evaluación de la calidad de servicio (Ortega y Suárez, 2009).

Con los objetivos que fueron planteados al inicio de este estudio se concluye que el ICCAIT tiene un nivel de consistencia interna alto; el instrumento está configurado por 10 factores (sala de espera; condiciones ambientales del centro; ubicación del centro; material; salas de tratamiento; atención al usuario; cualificación, coordinación y cercanía del personal al usuario; sugerencias, reclamaciones y quejas; derivación al centro e información técnica) y será una herramienta válida y pragmática que permitirá evaluar la calidad percibida en los CAIT por parte de los usuarios del servicio.

Para finalizar, a la luz de los resultados obtenidos en este trabajo puede afirmarse que este cuestionario constituye una herramienta de medida fiable y válida que permite explorar a partir de un único instrumento una información amplia de especial relevancia para los CAIT en particular y para la AT en general. El inventario puede resultar especialmente útil en un momento en el que la AT ha ampliado sus objetivos más allá de su papel tradicional de aplicar programas a los niños susceptibles de tratamiento, para ocuparse de una forma más íntegra de evaluar la calidad percibida por parte de los usuarios del servicio (Pegenaute, 2009). Sin embargo, como se ha comentado, a pesar de que cada vez es mayor el interés por la calidad de servicio en nuestro país, no se dispone de instrumentos validados que permitan evaluar la percepción de la calidad por parte de los clientes de la AT. Esto hace especialmente importante la disponibilidad de un cuestionario validado que permita a los profesionales de la AT evaluar la calidad percibida por parte de las familias usuarias del servicio.

Aunque no todo está hecho, estudios como éste son necesarios para constatar no solo los cambios positivos, sino también y con mayor énfasis los fallos y las deficiencias que todavía persisten en los servicios de AT. Conviene destacar que en la actualidad existen dos nuevos CAIT en la provincia de Málaga, por lo que debieran ser tenidos en cuenta a la hora de futuras investigaciones sobre el tema. De la misma manera que también debieran diseñarse instrumentos para evaluar la percepción de la calidad tanto por parte de los clientes internos como de los gerentes y/ directores de los CAIT.

Agradecimientos.-A todas las familias y personal que trabaja en los Centros de Atención Infantil Temprana participantes en este estudio, sin ellos la realización del mismo hubiese resultado tarea imposible.

Este trabajo forma parte de la investigación Observación de la interacción en deporte y actividad física: Avances técnicos y metodológicos en registros automatizados cualitativos-cuantitativos, que ha sido subvencionado por la Secretaría de Estado de Investigación, Desarrollo e Innovación del Ministerio de Economía y Competitividad [DEP2012-32124], durante el trienio 2012-2015. 


\section{Referencias}

Abad, F. J., Olea, F., Ponsoda, V. y García, C. (2011). Medición en ciencias sociales y de la salud. Madrid: Síntesis.

Anguera, M. T. (2003). La Metodología selectiva en el deporte. En A. Hernández Mendo (Coord.), Psicología del Deporte (vol. II) - Metodología (pp. 43-76). Buenos Aires: Tulio Guterman.

Arias-Astray, A. y Fernández-Ramírez, B. (1998). La encuesta como técnica de investigación social. En A.J. Rojas-Tejada, J.S. Fernández-Prados y C. Pérez-Meléndez (Eds.), Investigar mediante encuestas. Fundamentos teóricos $y$ aspectos prácticos (pp. 31-49). Madrid: Síntesis.

Arizcun, J., Gútiez, P. y Ruiz, E. (2006). Formación en Atención Temprana: Revisión histórica y estado de la cuestión. Recuperado de http://www.paidos.rediris.es/genysi/actividades/jornadas/xivjorp/xivGutiez.pdf

Barbarin, O. y Waslk, B. (2009). Handbook of child development and early education. New York: The Guilford Press.

Bugié, C. (2002). Centros de desarrollo infantil y Atención Temprana. Revista Neurología, 34(1), 139-143.

Cerqueira da Silva, S., Dessen, M. A. y Pérez-López, J. (2012). Evaluación de los servicios de atención a familias de niños con deficiencia. Perspectiva de familiares y profesionales brasileños. Anales de Psicología, 28 (3), 866-874. Doi.org/10.6018/analesps.28.3.130651.

Clark, A.L. y Watson, D. (1995). Constructing validity: Basic issues in objective scale development. Psychological Assessment, 7(3), 309-319.

Donabedian, A. (1988). The quality of care. How can it the assessed? The Journal American Medical Association, 260, 1743-1748.

García, F., Mirete, A., Marín, C. y Romero, L. (2008). Satisfacción del cliente familia en atención temprana: valoración de la importancia que otorgan a distintos aspectos del servicio. Siglo Cero, 39(3), 35-54.

García-Sánchez, F. A., Castellanos, P. y Mendieta, P. (1998): Definición de Atención Temprana y de sus vertientes de intervención desde un Modelo Integral. Revista de Atención Temprana, 1(2), 57-62.

García-Sánchez, F. A. (2002). Investigación en atención temprana. Revista Neurología, 34(1), 151-155.

Giné, C., Gràcia, M., Vilaseca, R., García-Díe, M. T. (2006). Repensar la atención temprana: propuestas para un desarrollo futuro. Infancia $y$ Aprendizaje, 29(3), 297-313.

Grupo de Atención Temprana (GAT) (2000). Libro blanco de la Atención Temprana. Madrid: Real Patronato sobre discapacidad. Ministerio de Sanidad, Política Social e Igualdad.

Grupo de Atención Temprana (GAT) (2005). Recomendaciones técnicas para el desarrollo de la Atención Temprana. Madrid: Real Patronato sobre Discapacidad. Ministerio de Trabajo y Asuntos Sociales.

Grupo de Atención Temprana (GAT) (2011). La realidad actual de la Atención Temprana en España. Madrid: Real Patronato sobre Discapacidad. Ministerio de Sanidad, Política Social e Igualdad.

Iversen, M. D, Shimmed, J. P., Ciacera, S. L. y Prabhakar, M. (2003). Creating a family-contered approach to early intervention services: perceptions of parents and profesionals. Pediatric Physical Therapy, 15, 23-31.

Kessel, F. \& Rosenfield, P. L. (2008). Prefacio de la nueva edición. En F. Kessel, P. L. Rosenfield \& N. B. Anderson (Eds.), Interdisciplinary research: Case studies from bealth and social science (pp. IX-XX). New York: Oxford University Press.

Kotler, P. (2000). Marketing Management. The Milennium Edition. New Jersey: Prentice Hall.

Martínez, R. (1996). Psicometría. Teoría de los test psicológicos y educativos. Madrid: Síntesis.

Medina i Mirapeix, F., De Oliveira Sousa, S. L., Lillo-Navarro, M. C., Montilla-Herrador, J., Escolar-Reina, P. y Gómez-Arnaldos, F. (2007). Percepción de los padres de la atención de fisioterapia recibida en centros de atención temprana. Revista de Atención Temprana, 10(1), 21-28.

Milla, M. G. y Mulas, F. (2002). Diagnóstico interdisciplinar en Atención Temprana. Revista Neurologia, 34(1), 139-143.

Morales-Sánchez, V. (2003). Evaluación Psicosocial de la calidad en los servicios municipales deportivos: aportaciones desde el análisis de la variabilidad. Málaga: SPICUM.
Morales-Sánchez, V., Hernández-Mendo, A y Blanco, A. (2009). Evaluación de la calidad en organizaciones deportivas: adaptación del modelo SERVQUAL. Revista de Psicología del deporte, 18(2), 137-150.

Morales-Sánchez, V. (2011). Evaluación de la calidad en organizaciones de servicios. En N. Balluerka, A. Gorostiaga, N. Lertxundi y L. Vozmediano (Coords.), XII Congreso de Metodología de las Ciencias Sociales y de la Salud. Libro de Resúmenes (p.54). San Sebastián: Asociación Española de Metodología de las Ciencias del Comportamiento y Universidad del País Vasco.

Muñiz, J., Fidalgo, A. M., García-Cueto, E., Martínez, R. y Moreno, R. (2005). Análisis de los items. Madrid: La Muralla, S.A

Ortega, M. C. y Suárez, M. G. (2009). Manual de evaluación de la calidad del servicio en enfermería. Estrategias para su aplicación ( $2^{\mathrm{a}}$ Ed.). México D.F.: Panamericana.

Pegenaute, F. (2009). Expectativas de los profesionales. En la Jornada de presentación del Manual de estándares de calidad y de acreditación de los centros de atención infantil temprana. Escuela Andaluza de Salud Pública. Granada, 19 de enero de 2009

Pereira-Silva, N. L. y Dessen, M. A. (2005). Intervenção precoce e familia: contribuições do modelo bioecológico de Bronfenbrenner. Em M. A. Dessen y A. L. Costa-Júnior (Dires.), A ciência do desenvolvimento bumano: tendências atuais e perspectivas futuras (pp. 152-157). Porto Alegre: Artmed.

Pérez-López, J. (2004). Modelos explicativos del desarrollo aplicados a la atención temprana. En J. Pérez-López y A. Brito de la Nuez (Eds.). Manual de Atención Temprana. (27-44). Madrid: Ediciones Pirámide.

Pérez-López, J. y Sánchez-Caravaca, J. (2008). Riesgo y tratamiento como factores responsables del progreso en los niños prematuros. En González-Pineda, J.A. y Núñez-Pérez, J.C. (Eds.). Psicología y educación: un lugar de encuentro, 1631-1640). Oviedo: Ediciones de la Universidad de Oviedo.

Pérez-López, J., Brito, A., Martínez-Fuentes, M.T., Díaz-Herrero, A., Sánchez-Caravaca, J., Fernández-Rego, F.J. y Casbas-Gómez, I. (2012). Las escalas Bayley BSID-I frente a BSDI-II como instrumento de evaluación en Atención Temprana. Anales de Psicología, 28(2), 484-489. Doi.org/10.6018/analesps.28.2.132481.

Perpiñán, S. (2009). Atención Temprana y familia. Cómo intervenir creando entornos competentes. Madrid: Narcea.

Pina, J. (2008). Análisis de un modelo de seguimiento en Atención Temprana. Universidad Complutense de Madrid: Tesis Doctoral.

Ponte, J. (2003a). La atención temprana en el ámbito de los servicios sociales. Minusval, Número especial monográfico sobre Atención Temprana, 79-84. Madrid: Imserso

Ponte, J. (2003b). Legislación y Atención Temprana. Nota sobre aspectos sociosanitarios. Boletin del Real Patronato sobre Discapacidad, 56, 5-19.

Ponte, J., Cardama, J., Arlanzón, J.L., Belda, J.C., González, T. y Vived, E. (2004). Guia de estándares de calidad en atención temprana. Madrid: Ministerio de Trabajo y Asuntos Sociales.

Romero-Galisteo, R. P. y Morales-Sánchez, V. (2010a). Calidad y atención temprana: breve revisión teórica. Lecturas: Educación Física y Deportes. Revista Digital, 145, Junio.

Romero-Galisteo, R. P. y Morales-Sánchez, V. (2010b). Atención temprana y calidad de servicio. Propuesta de una herramienta de medida. Lecturas: Educación Física y Deportes. Revista Digital, 145, Junio.

Romero-Galisteo, R. P., Morales-Sánchez, V. y Sánchez-Guerrero, E. (2011). Inventario de calidad en los centros de atención infantil temprana: Análisis factorial exploratorio. En N. Balluerka, A. Gorostiaga, N. Lertxundi y L. Vozmediano (Coords.), XII Congreso de Metodología de las Ciencias Sociales y de la Salud. Libro de Resúmenes (p.56). San Sebastián: Asociación Española de Metodología de las Ciencias del Comportamiento y Universidad del País Vasco.

Shonkoff, J. P. \& Phillips, D. A. (2000). Neurons to neighborhoods: The science of early child development. Washington, D.C.: National Academy Press.

Shonkoff, J. P. y Levitt, P. (2010). Neuroscience and the future of early childhood policy: Moving from why to what and how. Neuron, 67, 689692 . 
Tabachnik, B. G. y Fedell, L.S. (2007). Using Multivariate Statistic (5ª Ed.). Boston: Allyn and Bacon.

Tomás-Miguel, J.M. (1993). El uso de los modelos de ecuaciones estructurales y del análisis factorial confirmatorio en el análisis psicométrico de cuestionarios: una batería de seguridad laboral. Universidad de Valencia: Tesis doctoral.

Trianes, M. V., Blanca, M. J., Fernández-Baena, F. J., Escobar, M. Maldonado, E. F. y Muñoz, A. M. (2009). Evaluación del estrés infantil: Inventario Infantil de Estresores Cotidianos (IIEC). Psicothema, 21(4), 598603
Urbina Pérez, O., Pérez Pérez, M. y Martínez Sánchez, A. (2000). Flexibilidad organizativa y relación entre JIT y calidad total. Alta Dirección, 210(35), 74-84

Vanderven, K. (2008). Promoting Positive development in early cbildhood. New York: Springer.

Vegas, E. y Santibáñez, L. (2010). The promise of early childhood development in Latin America and the Caribbean. Washington: The World Bank.

Visauta, B., Martori, I. y Cañas, J. C. (2005). Análisis estadístico con SPSS para Windows. México: McGraw-Hill.

(Artículo recibido: 16-9-2012; revisado: 20-5-2013; aceptado: 31-5-2013) 


\section{Apéndice I}

\section{INVENTARIO DE CALIDAD EN LOS CENTROS DE ATENCIÓN INFANTIL TEMPRANA (ICCAIT)}

Estamos interesados en hacer una EVALUACIÓN DE LA CALIDAD EN LOS CENTROS DE ATENCIÓN INFANTIL TEMPRANA. Su opinión es muy importante para mejorar la calidad del servicio de nuestro centro. Por esta razón le agradeceremos que conteste al siguiente cuestionario.

Por favor, señale el número que más se aproxime a la valoración que usted quiera indicar, por ejemplo, si está "muy de acuerdo" señale la casilla $5 \mathrm{o}$, si por el contrario, está "nada de acuerdo" señale la casilla 1en los enunciados que aparecen a continuación. No existen respuestas malas o buenas, verdaderas o falsas, únicamente DESEAMOS CONOCER SU OPINIÓN SINCERA SOBRE NUESTRO SERVICIO. Los datos que aquí figuren serán confidenciales y únicamente serán utilizados con fines de investigación. Muchas gracias por su colaboración.

\begin{tabular}{|l|l|l|l|l|}
\hline Nada de acuerdo & & & & Muy de acuerdo \\
\hline 1 & 2 & 3 & 4 & 5 \\
\hline
\end{tabular}

\section{Escala 1: Relativa a las instalaciones}

1. El centro está bien ubicado geográficamente.

2. Es fácil llegar al centro en transporte público.

3. Las indicaciones para llegar al centro son suficientes.

4. El centro ha eliminado barreras arquitectónicas para facilitar su acceso (escaleras, escalones, etc.).

5. La limpieza del centro es adecuada.

6. La iluminación del centro es adecuada.

7. La ventilación de las instalaciones del centro le parece correcta.

8. La temperatura (calefacción, aire acondicionado) del centro le resulta agradable.

9. El centro cuenta con servicios (cambiador, inodoro, etc.) adaptados a las necesidades del usuario-

a/hijo-a.

10. Su centro cuenta con sala de espera.

11. Encuentra la sala de espera confortable.

12. La sala de espera cuenta con un número suficiente de sillas

13. La sala de espera cuenta con material lúdico (revistas, juguetes, cuentos, etc.) para hacerle más agradable su espera.

Escala 2: Relativa a las salas de tratamiento y material

14. El número de salas de tratamiento es adecuado.

15. El espacio de las salas de tratamiento es suficiente.

16. La temperatura de las salas de tratamiento es agradable.

17. La ventilación de las salas de tratamiento es correcta.

18. El material de trabajo que se utiliza en el centro es adecuado.

19. El centro cuenta con material suficiente para trabajar con el usuario-a/ hijo-a.

20. Los materiales están en buenas condiciones para su utilización.

21. El material que se utiliza en las salas de tratamiento es seguro.

22. El material de trabajo cumple con las condiciones higiénico-sanitarias (está limpio) para trabajar con él.

\section{Escala 3: Relativa a la atención del usuario}

23. La atención que recibe el usuario-a/hijo-a en el centro es adecuada.

24. El número de sesiones de tratamiento a la semana es suficiente.

25. El horario de atención se adapta a sus necesidades.

26. Se le ha facilitado un cambio de horario cuando así lo ha solicitado.

27. Se suele respetar el horario asignado por parte del centro.

28. Cuando su hijo-a/usuario-a mejora o empeora, el centro le cambia la frecuencia de tratamiento.

29. Cuando, por cualquier motivo, le anulan una cita, le avisan con tiempo suficiente.

Escala 4: Relativa al personal especializado

30. El personal especializado posee los conocimientos necesarios.

31. El personal especializado es accesible. 
32. El personal especializado está cuando se le necesita.

33. El personal especializado resulta cercano en el trato.

34. Piensa que el usuario-a/ hijo-a se encuentra cómodo con el personal del centro.

35. Valora usted las aportaciones e iniciativas del personal especializado.

36. El personal especializado se coordina entre sí para mejorar y completar la atención del usuario-a/hijo-a.

37. El personal especializado sabe adaptar las tareas a las necesidades del usuario-a/hijo-a.

Escala 5: Relativa a la información general

38. Ha conocido el centro por información en el colegio.

39. Ha conocido el centro por derivación de Asuntos Sociales.

40. El centro posee buzón de sugerencias.

41. El centro cuenta con hojas de reclamaciones.

42. Considera necesaria la existencia de una persona a la que dirigir sus quejas y/o sugerencias.

Escala 6: Relativa a la información técnica

43. Las actividades que se realizan en el centro con el usuario-a/hijo-a le parecen las adecuadas.

44. Las actividades que le han propuesto en el centro para trabajar con el usuario-a/hijo-a en casa le resultaron cercanas a sus posibilidades.

45. La información recibida al inicio del tratamiento es coherente con las tareas desempeñadas posterior mente.

46. Recibe indicaciones/programas por parte del centro para trabajar con el usuario-a/hijo-a.

47. Recibe algún tipo de informe (oral/escrito) sobre la progresión del usuario-a/hijo-a.

48. La información que recibe sobre su hijo-a/usuario/a es clara.

\begin{tabular}{|l|l|l|}
\hline \multicolumn{2}{|l|}{} & \multicolumn{2}{l|}{$\begin{array}{l}\text { Parentesco con el/la pa- } \\
\text { ciente } \\
\text { (señalar con una X) }\end{array}$} \\
\hline Nombre del Centro: & Madre: & \\
\hline Eénero: H (1. Hombre) M (2. Mujer) & Padre: & \\
\hline Lugar de Nacimiento: & Tía: & \\
\hline Sin estudios: & Tío: & \\
\hline Estudios primarios (EGB, Primaria, Secundaria, etc.): & Abuela: & \\
\hline Estudios medios (BUP, ESO, FP, Bachiller, etc.) & Abuelo: & \\
\hline Estudios Superiores (Universitarios): & Cuidadora: & \\
\hline Estudios Posgrados (Doctorados y Masters): & Cuidador: & \\
\hline Otros (indíquelo): & Otros (indíquelo): \\
\hline ¿Cuánto tiempo lleva acudiendo a este centro? & \\
\hline ¿Anteriormente, ha acudido a otro centro de atención infantil temprana? & \\
\hline
\end{tabular}

Si tiene alguna OBSERVACIÓN y/o SUGERENCIA que realizar, por favor, escríbala a continuación: 